\title{
Bjorken hydrodynamics and gradual freeze out
}

\author{
Volodymyr Magas* \\ Departament d'Estructura i Constituents de la Matéria, \\ Universitat de Barcelona, Diagonal 647, 08028 Barcelona, Spain \\ E-mail: vladimir@ecm.ub.es
}

\section{László P. Csernai}

Theoretical and Energy Physics Unit, University of Bergen,

Allegaten 55, 5007 Bergen, Norway \&

MTA-KFKI, Research Inst of Particle and Nuclear Physics,

H-1525 Budapest 114, P.O.Box 49, Hungary

E-mail: csernai@ift.uib.no

\section{Etele Molnár}

Frankfurt Institute for Advanced Studies, Johann Wolfgang Goethe University

Max-von-Laue-Str. 1, 60438 Frankfurt am Main, Germany

E-mail: molnarefias.uni-frankfurt.de

The freeze out of the expanding systems, created in relativistic heavy ion collisions, is discussed. We combine kinetic freeze out equations with Bjorken type system expansion into a unified model. The important feature of the proposed scenario is that physical freeze out is completely finished in a finite time, which can be varied from 0 (freeze out hypersurface) to $\infty$. The dependence of the post freeze out distribution function on the freeze out time will be studied. Model allows analytical analyses for the simplest systems such as pion gas. We shall see that the basic freeze out features, pointed out in the earlier works, are not smeared out by the expansion of the system. The entropy evolution in such a scenario is also studied.

Critical Point and Onset of Deconfinement

July 3-6 2006

Florence, Italy

\footnotetext{
${ }^{*}$ Speaker.
} 


\section{Introduction}

In the ultrarelativistic heavy ion collisions at RHIC the total number of the produced particles exceeds several thousands, therefore one can expect that the produced system behaves as a "matter" and generates collective effects. Indeed strong collective flow patterns have been measured at RHIC, which suggests that the hydrodynamical models are well justified during the intermediate stages of the reaction: from the time when local equilibrium is reached until the freeze out (FO), when the hydrodynamical description breaks down. During this FO stage, the matter becomes so dilute and cold that particles stop interacting and stream towards the detectors freely, their momentum distribution freezes out. The FO stage is essentially the last part of a collision process and the main source for observables.

In simulations FO is usually described in two extreme ways: A) FO on a hypersurface with zero thickness, B) FO described by volume emission model or hadron cascade, which in principle requires an infinite time and space for a complete FO. At first glance it seems that one can avoid troubles with FO modeling using hydro+cascade two module model [1], since in hadron cascades gradual FO is realized automatically. However, in a such a scenario there is an uncertain point, actually uncertain hypersurface, where one switches from hydrodynamical to kinetic modeling. First of all it is not clear how to determine such a hypersurface. This hypersurface in general may have both time-like and space-like parts. Mathematically this problem is very similar to hydro to FO phase transition on the infinitely narrow FO hypersurface, therefore for example all the problems discussed for FO on the hypersurface with space-like normal vectors will take place here. Another complication is that while for the post FO domain we have mixture of non-interacting ideal gases, now for the hadron cascade we should generate distributions for the interacting hadronic gas of all possible species, as a starting point for the further cascade evolution. The volume emission models are based on the kinetic equations [2,3] defining the evolution of the distribution functions, and therefore these also require to generate initial distribution functions for the interacting hadronic species on some hypersurface.

In this paper we present a simple kinetic FO model, which describes the freeze out of particles from a Bjorken expanding fireball [4]. The important feature of the proposed scenario is that physical freeze out is completely finished in a finite time, which can be varied from 0 (freeze out hypersurface) to $\infty$. In the other words our freeze out happens in a layer, i.e. in a domain restricted by two parallel hypersurfaces $\tau=\tau_{1}$ and $\tau=\tau_{1}+L$ ( $\tau$ is the proper time). The present model describes simultaneously freeze out and Bjorken expansion, and thus, it is more physical extension of the oversimplified FO models without expansion [3, 5, 6, 7, 8]. In Ref. [6] authors have also adopted kinetic gradual FO model to Bjorken geometry, but combined it with Bjorken expansion on the consequent, not on the parallel basis: system expands according to Bjorken hydro scenario, but when it reaches beginning of the FO process system stops expansion and gradually freezes out in a fixed volume. It was shown in [6] that although such a model allows to obtain analytical results, it is not physical, the simultaneous modeling of expansion and freeze out is required in order to avoid decreasing of the total entropy. And now we propose such a generalized model. 


\section{Finite layer freeze out description}

Let us briefly review gradual FO model, which we are going generalize including expansion. Many building blocks of the model are Lorentz invariant and can be applied to both time-like and space-like FO layers, so at the beginning we will write these in general way. Starting from the Boltzmann Transport Equation, introducing two components of the distribution function, $f$ : the interacting, $f^{i}$, and the frozen out, $f^{f}$ ones, $\left(f=f^{i}+f^{f}\right)$, and assuming that FO is a directed process (i.e. neglecting the gradients of the distribution functions in the directions perpendicular to the FO direction comparing to that in the FO direction) we can obtain the following system of the equations [7,9]:

$$
\frac{d f^{i}}{d s}=-\frac{P_{e s c}}{\tau_{F O}} f^{i}+\frac{f_{e q}(s)-f^{i}}{\tau_{t h}}, \quad \frac{d f^{f}}{d s}=\frac{P_{e s c}}{\tau_{F O}} f^{i} .
$$

The FO direction is defined by the unit vector $d \sigma_{\mu}$. FO happens in a layer of given thickness $L$ with two parallel boundary hypersurfaces perpendicular to $d \sigma_{\mu}$, and $s=d \sigma_{\mu} x^{\mu}$ is a variable in the FO direction. We work in the reference frame of the front, where $d \sigma_{\mu}$ is either $(1,0,0,0)$ for the time-like FO, or $(0,1,0,0)$ for the space-like FO. The $\tau_{F O}$ is some characteristic length scale, like mean free path or mean collision time for time-like FO. The rethermalization of the interacting component is taken into account via the relaxation time approximation, where $f_{i}$ approaches the equilibrated Jüttner distribution, $f_{e q}(s)$, with a relaxation length/time, $\tau_{t h}$. The system (2.1) can be solved semi-analytically in the fast rethermalization limit [7].

The basis of the model, i.e. the invariant escape rate within the FO layer of the thickness $L$, for both time-like and space-like normal vectors is given as (see Refs. $[7,8,10]$ for more details)

$$
P_{e s c}=\left(\frac{L}{L-s}\right)\left(\frac{p^{\mu} d \sigma_{\mu}}{p^{\mu} u_{\mu}}\right) \Theta\left(p^{\mu} d \sigma_{\mu}\right)
$$

where $p^{\mu}$ is a particle four-momentum, $u^{\mu}$ is the flow velocity. In fact the model based on the escape rate (2.2) is a generalization of a simple kinetic models studied in Refs. [3, 5, 6], which can be restored in the $L \rightarrow \infty$ limit. Here we will concentrate on the time-like case only, where the above $\Theta$ function is unity.

The important feature of the escape rate in the form (2.2) is that physical freeze out is completely finished when $s=L$, i.e. it requires finite space/time. Furthermore, now we can vary this layer thickness, $L$, from 0 (freeze out hypersurface) to $\infty$ and study how the post FO distribution depends on the layer thickness. Interesting and unexpected result was found in $[7,8,10]$, for both space-like and time-like FO layers, namely that if $L$ is large enough, at least several $\tau_{F O}$, then post FO distribution gets some universal form, independent on the layer thickness.

Simple semianalytically solvable FO models studied in $[3,5,6,7,8]$ are missing an important ingredient - the expansion of the freezing out system. The open question is whether the discussed above features of the FO will survive if the system expansion is included. In this work we are going to build a model, which includes both gradual FO and Bjorken-like expansion of the system, and answer this question.

\section{Bjorken expansion with gradual freeze out}

Let us first remind the reader the basics of the Bjorken model 3.1. Bjorken model is one- 
dimensional in the same sense as discussed before eq. (2.1) - only the proper time, $\tau=\sqrt{t^{2}-x^{2}}$, gradients are considered. Here the reference frame of the front, $d \sigma^{\mu}=(1,0,0,0)$, is the same as the local rest frame, $u^{\mu}=(1,0,0,0)$. The evolution of the energy density and baryon density is given by the following equations:

$$
\frac{d e}{d \tau}=-\frac{e+P}{\tau}, \quad \frac{d n}{d \tau}=-\frac{n}{\tau},
$$

where $P$ is the pressure. The initial conditions are given at some $\tau=\tau_{0}: e\left(\tau_{0}\right)=e_{0}, n\left(\tau_{0}\right)=n_{0}$. This system can be easily solved:

$$
e(\tau)=e_{0}\left(\frac{\tau_{0}}{\tau}\right)^{1+c_{o}^{2}}, \quad n(\tau)=n_{0}\left(\frac{\tau_{0}}{\tau}\right)
$$

where $P=c_{o}^{2} e$ is the equation of state (EoS) in general form.

It is important to remember that if we want to have a finite volume fireball, we need to put some boarders on the system. Here we assume that our system, described by the Bjorken model, is situated in the spacial domain $|\eta| \leq \eta_{R}$ or what is the same $|z| \leq z_{R}(\tau)=\tau \sinh \eta_{R}\left(\eta=\frac{1}{2} \ln \left(\frac{t+z}{t-z}\right)\right.$ is pseudorapidity). Within this boarder system is uniform along $\tau=$ const hyperbolas; outside we have vacuum with zero energy and baryon densities as well as pressure. Thus, we have a jump, a discontinuity on the border, which stays there during all the evolution. Certainly, to prevent matter expansion through such a border (due to strong pressure gradient) some work is done on the border surface [11]. One can find about it as about putting some effective pressure to the vacuum, exactly the one which would remove discontinuity, then this the work done by the expanding system against some pressure. As system expends the volume of the fireball increases as

$$
V(\tau)=2 A_{x y} \sinh \eta_{R} \tau
$$

where $A_{x y}$ is the transverse area of the system. Work done by the expanding system, $W$, is given by

$$
d W=P d V \quad \Rightarrow \quad W(\tau)=e_{0} V_{0}\left(1-\left(\frac{\tau_{0}}{\tau}\right)^{c_{o}^{2}}\right)
$$

where we denote $V_{0}=V\left(\tau_{0}\right)$. One can easily check then the energy conservation:

$$
E_{\text {tot }}=e(\tau) V(\tau)+W(\tau)=e_{0} V_{0}=\text { const } .
$$

Applying our FO model to such a system, we obtain:

$$
\begin{gathered}
d f^{i}\left(\tau^{\prime}\right)=-\frac{d \tau^{\prime}}{\tau_{F O}} \frac{L}{L-\tau^{\prime}} f^{i}\left(\tau^{\prime}\right)+\frac{d \tau^{\prime}}{\tau_{t h}}\left[f_{e q}\left(\tau^{\prime}\right)-f^{i}\left(\tau^{\prime}\right)\right], \\
d f^{f}\left(\tau^{\prime}\right)=+\frac{d \tau^{\prime}}{\tau_{F O}} \frac{L}{L-\tau^{\prime}} f^{i}\left(\tau^{\prime}\right),
\end{gathered}
$$

where FO begins at $\tau=\tau_{1}$ and $\tau^{\prime}=\tau-\tau_{1}$. Taking the fast rethermalization limit, similarly to what is done in [6], we can obtain simplified equations for $f^{i}$, which is a thermal distribution $f^{i}(\tau)=f_{e q}(\tau), f^{f}$ as well as for $e^{i}, n^{i}$ and $e^{f}, n^{f}$ :

$$
\frac{d e^{i}}{d \tau^{\prime}}=-\frac{e^{i}}{\tau_{F O}} \frac{L}{L-\tau^{\prime}}, \quad \frac{d n^{i}}{d \tau^{\prime}}=-\frac{n^{i}}{\tau_{F O}} \frac{L}{L-\tau^{\prime}},
$$




$$
\frac{d e^{f}}{d \tau^{\prime}}=+\frac{e^{i}}{\tau_{F O}} \frac{L}{L-\tau^{\prime}}, \quad \frac{d n^{f}}{d \tau^{\prime}}=+\frac{n^{i}}{\tau_{F O}} \frac{L}{L-\tau^{\prime}} .
$$

Now the idea is to create a system of equations which would describe a fireball which simultaneously expands and freezes out. Let us put our two components $\left(e=e^{i}+e^{f}\right)$ into the first equation of (3.1) and do some simple algebra:

$$
\frac{d e^{i}}{d \tau}+\frac{d e^{f}}{d \tau}=-\frac{e^{i}+P^{i}}{\tau}-\frac{e^{f}}{\tau}-\frac{e^{i}}{\tau_{F O}} \frac{L}{L-\tau^{\prime}}+\frac{e^{i}}{\tau_{F O}} \frac{L}{L-\tau^{\prime}},
$$

where last two terms add up to zero; the free component, of course, has no pressure. So far our eq. (3.10) is completely identical to the first equation of (3.1). Our assumption is that our system evolves in such a way that eq. (3.10) is satisfied as a system of two separate equations for interacting and free components [12]:

$$
\begin{gathered}
\frac{d e^{i}}{d \tau}=-\frac{e^{i}+P^{i}}{\tau}-\frac{e^{i}}{\tau_{F O}} \frac{L}{L+\tau_{1}-\tau}, \\
\frac{d e^{f}}{d \tau}=-\frac{e^{f}}{\tau}+\frac{e^{i}}{\tau_{F O}} \frac{L}{L+\tau_{1}-\tau} .
\end{gathered}
$$

Similarly we can obtain equations for baryon density [12]:

$$
\begin{aligned}
& \frac{d n^{i}}{d \tau}=-\frac{n^{i}}{\tau}-\frac{n^{i}}{\tau_{F O}} \frac{L}{L+\tau_{1}-\tau}, \\
& \frac{d n^{f}}{d \tau}=-\frac{n^{f}}{\tau}+\frac{n^{i}}{\tau_{F O}} \frac{L}{L+\tau_{1}-\tau} .
\end{aligned}
$$

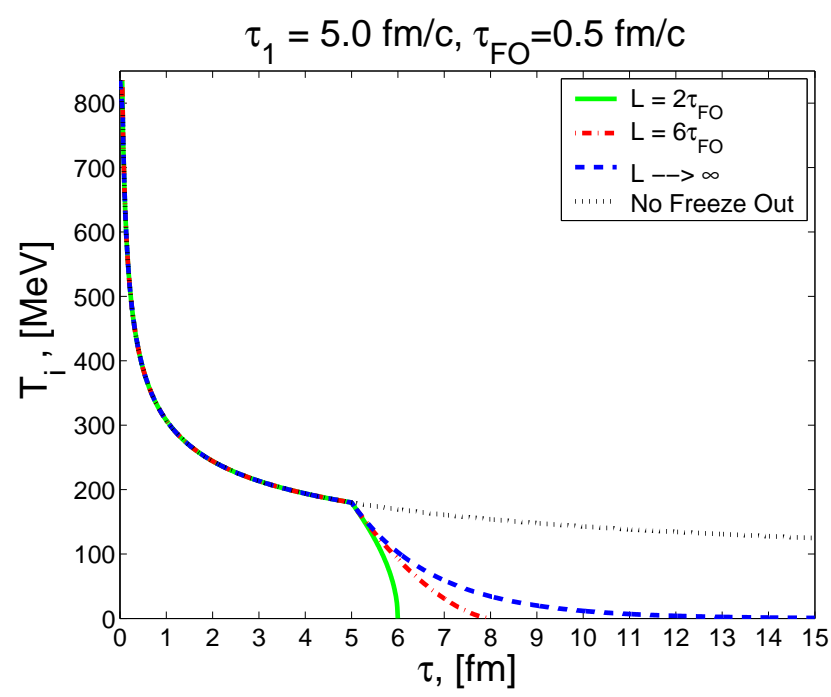

Figure 1: Evolution of the temperature of the interacting matter for different FO layers. $T_{i}\left(\tau_{0}=0.05 \mathrm{fm}\right)=$ $835 \mathrm{MeV}, T_{F O}=180 \mathrm{MeV}$. "No Freeze Out" means that we used standard Bjorken hydrodynamics even in phase II.

Thus, finally, we have the following simple model of fireball created in relativistic heavy ion collision. 
Initial state, $\tau=\tau_{0} e_{0}, n_{0}$

Phase I, Pure Bjorken hydrodynamics, $\tau_{0} \leq \tau \leq \tau_{1}$

$$
e(\tau)=e_{0}\left(\frac{\tau_{0}}{\tau}\right)^{1+c_{o}^{2}}, \quad n(\tau)=n_{0}\left(\frac{\tau_{0}}{\tau}\right)
$$

Phase II, Bjorken expansion and gradual FO, $\tau_{1} \leq \tau \leq \tau_{1}+L$

Solving Eqs. $(3.11,3.13)$ we obtain:

$$
\begin{gathered}
e^{i}(\tau)=e_{0}\left(\frac{\tau_{0}}{\tau}\right)^{1+c_{o}^{2}}\left(\frac{L+\tau_{1}-\tau}{L}\right)^{L / \tau_{F O}}, \\
n^{i}(\tau)=n_{0}\left(\frac{\tau_{0}}{\tau}\right)\left(\frac{L+\tau_{1}-\tau}{L}\right)^{L / \tau_{F O}} .
\end{gathered}
$$

The difference with respect to the pure Bjorken solution (3.15) is in the last multiplier, and we see that, as expected, the interacting component completely disappear then $\tau$ reaches $\tau=L+\tau_{1}$.

With these last equations we have completely determined evolution of the interacting component [12]. Knowing $e^{i}(\tau)$ and EoS we can find temperature, $T_{i}(\tau)$. Due to symmetry of the system $u_{i}^{\mu}(\tau)=u^{\mu}\left(\tau_{0}\right)=(1,0,0,0)$. Finally, $f^{i}(\tau)$ is a thermal distribution with given $T_{i}(\tau), n^{i}(\tau), u_{i}^{\mu}(\tau)$.

However for us the more interesting is free component, which is the source of the observables. Eqs. $(3.12,3.14)$ give us the evolution of the $e_{f}$ and $n_{f}$, and one can easily check that these two equations are equivalent to the following equation on the distribution function:

$$
\frac{d f^{f}}{d \tau}=-\frac{f^{f}}{\tau}+\frac{f^{i}}{\tau_{F O}} \frac{L}{L+\tau_{1}-\tau}
$$

The measured post FO spectra are given by $f^{f}\left(L+\tau_{1}\right)$.

\section{Solution of the model for massless boson gas}

Aiming for a qualitative illustration of the FO process we show below the results for the massless ideal gas without conserved charges with Jüttner equilibrated distribution [13]:

$$
\begin{gathered}
f^{i}(\tau, \mathbf{p})=\frac{1}{(2 \pi)^{3}} e^{-|\mathbf{p}| / T_{i}(\tau)}, \\
P^{i}=e^{i} / 3, \quad e^{i}=\frac{3}{\pi^{2}} T_{i}^{4} .
\end{gathered}
$$

We have taken the following values of the parameters: $\eta_{R}=5.4^{1}, A_{x y}=\pi R_{A u}^{2}$, where $R_{A u}=7.685$ $\mathrm{fm}$ is the $\mathrm{Au}$ radius, $\tau_{0}=0.05 \mathrm{fm}, T_{i}\left(\tau_{0}\right)=835 \mathrm{MeV} ; \tau_{1}=5 \mathrm{fm}, T_{i}\left(\tau_{1}\right)=T_{F O}=180 \mathrm{MeV}$; $\tau_{F O}=0.5 \mathrm{fm}$ and we present results for different values of FO time $L$.

Fig. 1 shows the evolution of the temperature of the interacting matter $\left(T_{i}(\tau)=\frac{\pi^{2}}{3}\left(e^{i}\right)^{1 / 4}\right)$, and Figs. 2 present the evolution of the energy densities for the interacting and free components (these satisfy the energy conservation $\left.V(\tau)\left(e^{i}(\tau)+e^{f}(\tau)\right)+W(\tau)=e_{0} V_{0}\right)$. We can analytically solve eqs. (3.11) and (3.12) also in $L \rightarrow \infty$ limit:

$$
e^{i}(\tau)=e_{0}\left(\frac{\tau_{0}}{\tau}\right)^{1+c_{o}^{2}} e^{-\frac{\tau_{1}-\tau}{\tau_{F O}}}
$$



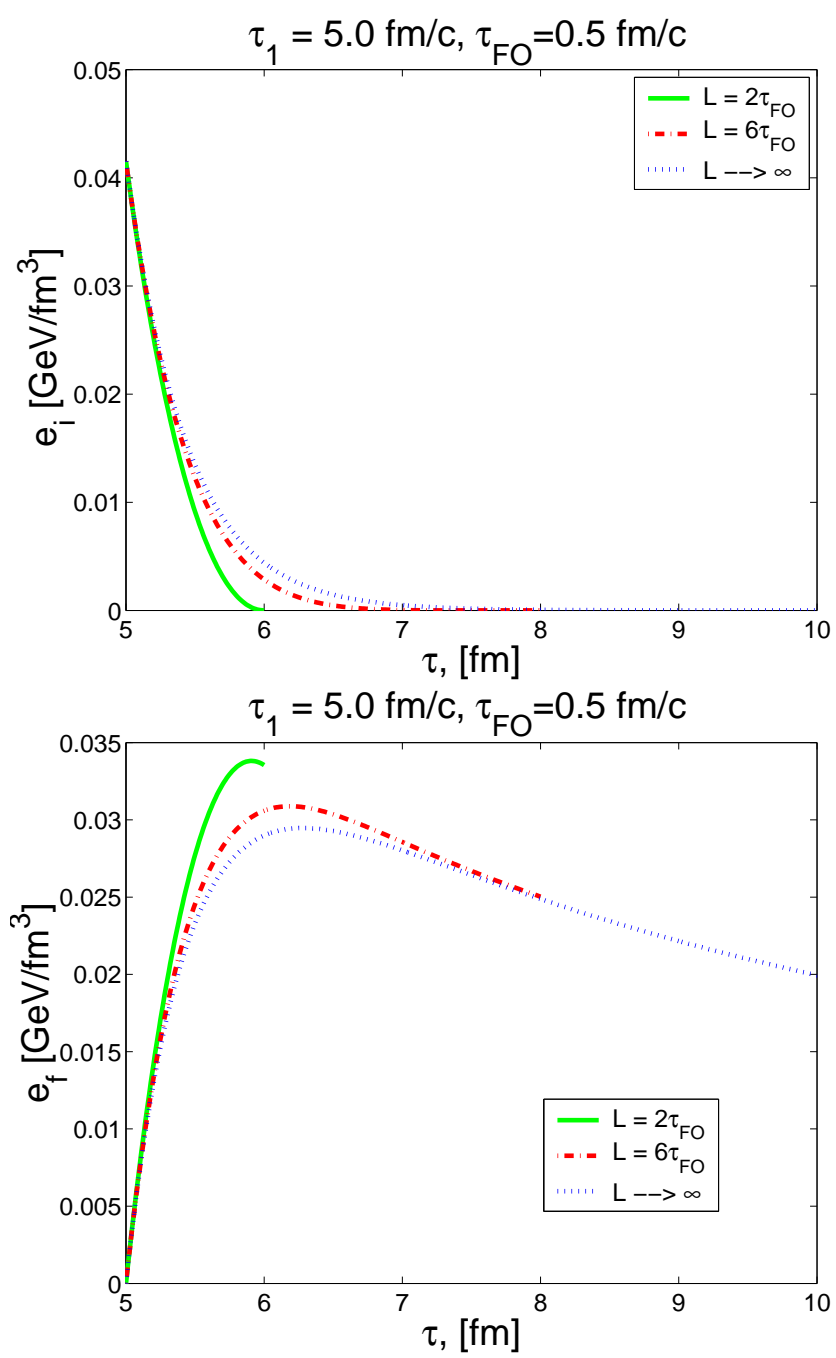

Figure 2: Evolution of the energy densities for the interacting (upper plot) and free (lower plot) components for different FO layers during phase II. $T_{i}\left(\tau_{0}=0.05 \mathrm{fm}\right)=835 \mathrm{MeV}, T_{F O}=180 \mathrm{MeV}$.

what is also shown in the Figs 1 and 2.

As it was already shown in $[6,8]$ the final post FO particle distributions, shown on Fig. 3, are non-equilibrated distributions, which deviate from thermal ones particularly in the low momentum region. By introducing and varying the thickness of the FO layer, $L$, we are strongly affecting the evolution of the interacting component, see Fig. 1, but the final post FO distribution shows strong universality: for $L>2 \tau_{F O}$ it already looks very close to that for an infinitely long FO calculations - see Fig. 3. The inclusion of the expansion into our consideration does not smear out this very important feature of FO. "No Expansion" curve is given by the analytical expression:

$$
f^{f}(\mathbf{p})=-\frac{4}{(2 \pi)^{3}} E i\left(-\frac{|\mathbf{p}|}{T_{F O}}\right)
$$

\footnotetext{
${ }^{1}$ We choose the limiting pseudorapidity for our fireball to be equal to the rapidity of the colliding nuclei in Lab frame at RHIC for $\sqrt{s}=100 \mathrm{GeV} /$ nucl.
} 

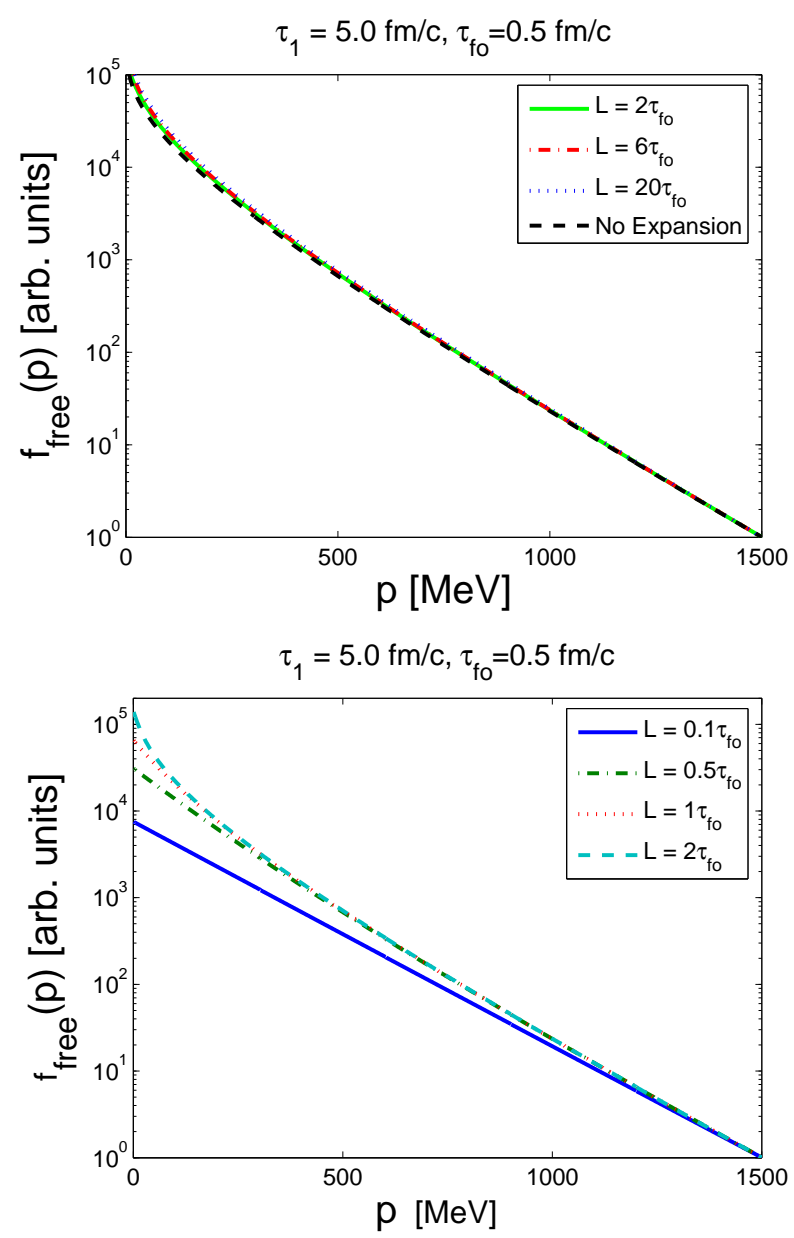

Figure 3: Final post FO distribution for different FO layers as a function of the momentum in the FO direction, $p=p^{x}$ in our case $\left(p^{y}=p^{z}=0\right)$. The initial conditions are specified in the text. "No Expansion" curve is given by the analytical expression from Ref. [6], eq. (4.4).

from Ref. [6], where gradual freeze out and Bjorken expansion were combined on the consequent, not on the parallel basis: system expands according to Bjorken hydro scenario, but when it reaches beginning of the FO process system stops expansion and gradually freezes out in a fixed volume. Although such a scenario is unrealistic ${ }^{2}$, but due to this universality of the final post FO distribution, it gives rather good description of the final spectrum.

If we make $L$ very small, $L<0.5 \tau_{F O}$, then the final post FO distribution is practically a thermal distribution: Fig. 3 lower plot. Here the FO process does not have enough time to distort the thermal shape of the distribution. More results can be found in [12].

In our opinion these results may justify the use of FO hypersurface in hydrodynamical models for heavy ion collisions, but with a proper non-thermal post FO distributions. If the FO layer is thick enough, say $L>2 \tau_{F O}$, then it doesn't matter how thick was FO layer, we do not need to model the FO dynamics in details. Once we have a good parameterization of the post FO spectrum

\footnotetext{
${ }^{2}$ The necessity of the expansion was proven in Ref. [6] based on non-decreasing entropy condition.
} 
(still asymmetric, non-thermal), for example analytical post FO distribution obtained in Ref. [6] (see Fig. 3), then the parameters of this distribution can be found from the conservation laws, as it is usually done for sharp FO, with some volume scaling factor to effectively account for the expansion during FO.
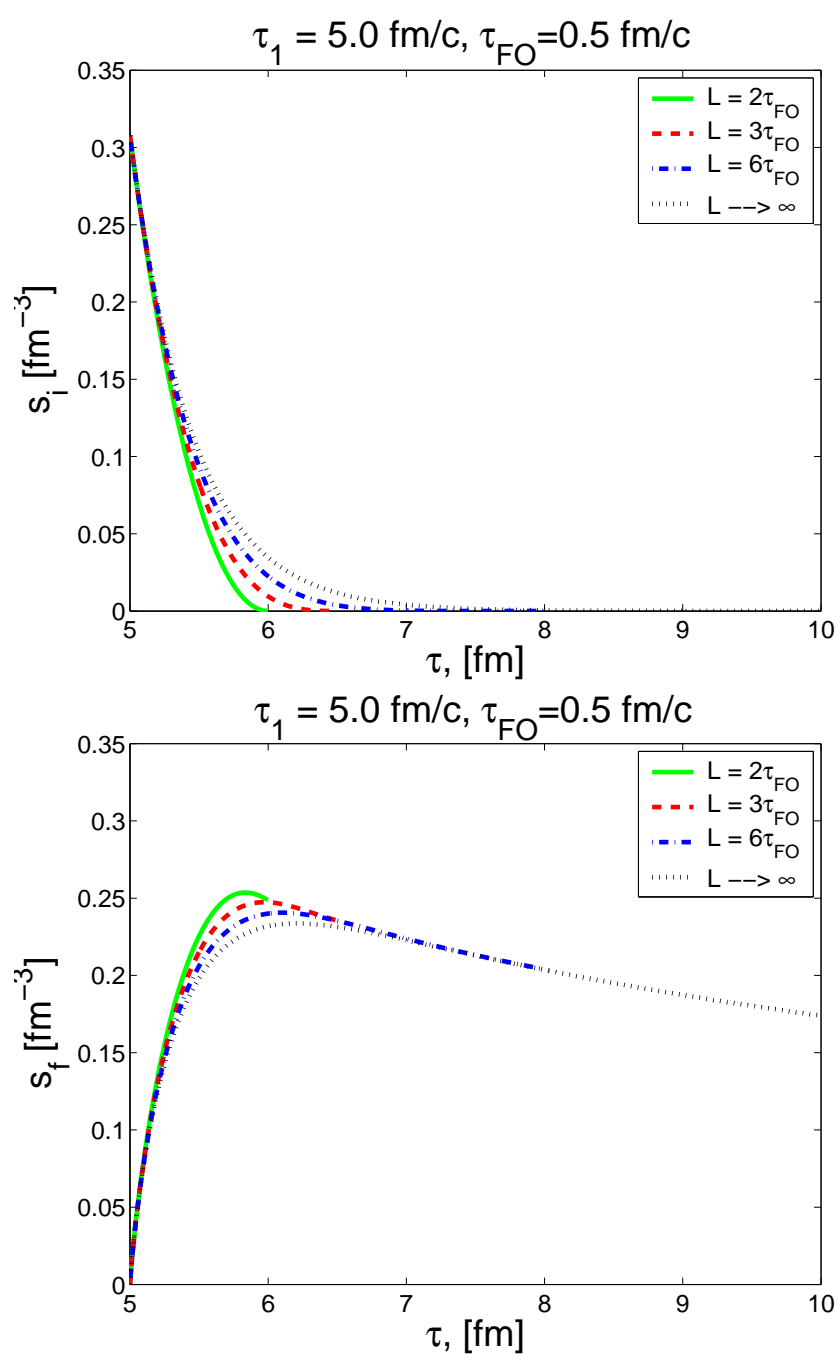

Figure 4: Evolution of the entropy densities for the interacting (upper plot) and free (lower plot) components for different FO layers during phase II. The initial conditions are specified in the text.

It is important to always check the non-decreasing entropy condition $[12,14]$ to see whether such a process is physically possible. Figs. 2 present the evolution of the entropy densities for the interacting and free components, but the total entropy, $S(\tau)$, is not a sum of these two densities multiplied by volume of the system, but it should be calculated based on the full distribution function, $f(\mathbf{p})=f^{i}(\mathbf{p})+f^{f}(\mathbf{p})$ :

$$
s(\tau)=\int d^{3} p f(\tau)\left[1-\ln \left((2 \pi)^{3} f(\tau)\right)\right], \quad S(\tau)=s(\tau) V(\tau) .
$$

Evolution of the total entropy versus initial entropy, $S_{0}=S\left(\tau_{0}\right)$, is shown on Fig. 5. As expected during pure Bjorken phase total entropy is constant, but then FO starts the total entropy makes a 
wiggle: first decreasing and then increasing until FO is finished. From this figure we can make two very important conclusions. The first one is that long gradual freeze out produces entropy. In our simulation for the FO layer as thick as $2.5-5 \mathrm{fm} / \mathrm{c}$ the total entropy production reaches $5-10 \%$. The second important conclusion is that thin FO layers, with $L \leq 3 \tau_{F O}$ are physically forbidden, because this would lead to entropy decrease. And this put as into the $L$ region, where the universality of the final post FO distribution really takes place.

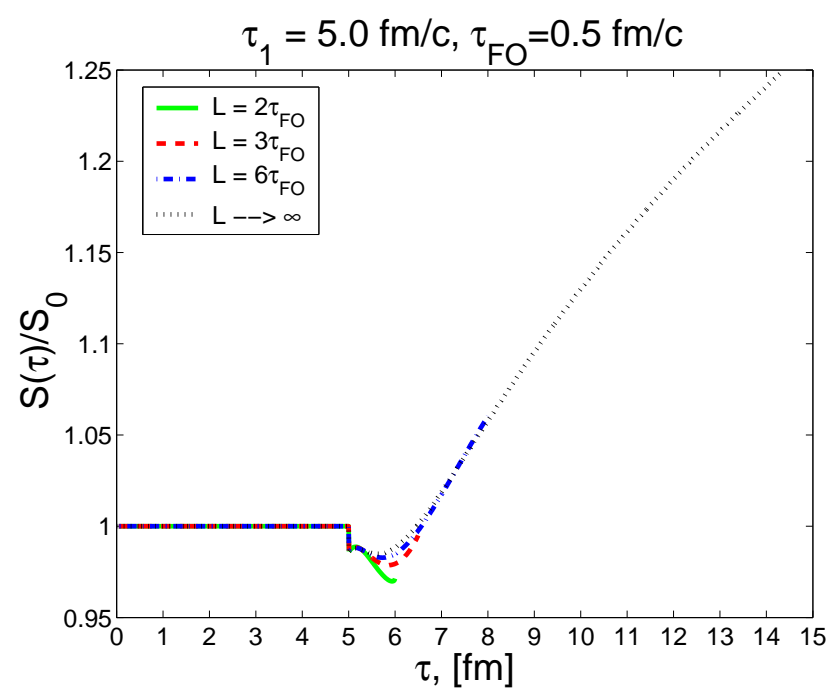

Figure 5: Evolution of the total entropy for different FO layers. The initial conditions are specified in the text.

\section{Conclusions}

In this paper we presented FO model with Bjorken like expansion in our FO model, in contrast to the older versions $[3,5,6,7,8]$, which allows us to study FO in a layer of any thickness, $L$, from 0 to $\infty$. Another good feature of the proposed model is that it connects the pre FO hydrodynamical quantities, like energy density, $e$, baryon density, $n$, with post FO distribution function in a relatively simple way, and furthermore allows analytical analyses for the simplest systems such as pion gas.

The results show that the inclusion of the expansion into FO model, although strongly affects the evolution of the interacting component, does not smear out the universality of the final post FO distribution, observed already in Refs. [6, 7, 8]: for $L>2 \tau_{F O}$ it already looks very close to that for an infinitely long FO calculations - see Fig. 3. Furthermore the non-decreasing entropy condition tell us that only relatively thick FO layers, $L \geq 3 \tau_{F O}$, are physically allowed. In one of the older works, Ref. [6], authors obtained the analytical expression for the final post FO spectrum, which is in a good agreement with the results obtained in more advanced model presented here. Thus, our consideration advises to try to fit experimental data using non-equilibrated distributions, given by eq. (4.4).

Another conclusion of this work, stressing once again the importance to always check the non-decreasing entropy condition [12,14], is that long gradual freeze out may produce substantial amount of entropy, as shown on Fig. 5. 


\section{References}

[1] D. Teaney et al., Phys. Rev. Lett. 83 (1999) 4951; S.A. Bass, A. Dumitru, Phys. Rev. C61 (2000) 064909; C. Nonaka, S.A. Bass, Nucl. Phys. A774 (2006) 873.

[2] F. Grassi et al., Phys. Lett B355 (1995) 9; Z. Phys. C73 (1996) 153; Yu.M. Sinyukov et al., Phys. Rev. Lett. 89 (2002) 052301.

[3] V.K. Magas et al., Heavy Ion Phys. 9 (1999) 193.

[4] J. D. Bjorken, Phys. Rev. D 27 (1983) 140.

[5] Cs. Anderlik et al., Phys. Rev. C59 (1999) 388; Phys. Lett. B459 (1999) 33; V.K. Magas et al., Nucl. Phys. A661 (1999) 596.

[6] V.K. Magas et al., Eur. Phys. J. C30 (2003) 255.

[7] E. Molnár et al., Phys. Rev. C74 (2006) 024907, V.K. Magas et al., nucl-th/0510066.

[8] E. Molnár et al., nucl-th/0503048.

[9] V.K. Magas et al., Nucl. Phys. A749 (2005) 202; L.P. Csernai et al., hep-ph/0406082; Eur. Phys. J. A25 (2005) 65.

[10] L.P. Csernai et al., hep-ph/0401005; E. Molnár et al., nucl-th/0510062.

[11] K. J. Eskola, K. Kajantie and P. V. Ruuskanen, Eur. Phys. J. C 1 (1998) 627.

[12] V.K. Magas, talk at the IVth International Conference on Quarks and Nuclear Physics QNP06, Madrid, Spain, June 5-10, 2006; E. Molnár et al., in preparation.

[13] F. Jüttner, Ann. Phys. Chem. 34, 856 (1911).

[14] Cs. Anderlik et al., Phys. Rev. C59 (1999) 3309. 